\title{
The LSST Real-Time Active Optics System
}

\author{
Sandrine J. Thomas ${ }^{\mathrm{a}}$, Bo Xin ${ }^{\mathrm{a}}$, Te-Wei Tsai ${ }^{\mathrm{a}}$, Christopher Contaxis ${ }^{\mathrm{a}}$, Chuck Claver ${ }^{\mathrm{a}}$, Paul \\ Lotz $^{\mathrm{a}}$, and Doug Neill ${ }^{\mathrm{a}}$ \\ ${ }^{a}$ LSST, 950 N. Cherry Av., Tucson, USA
}

\begin{abstract}
The Large Synoptic Survey Telescope is an $8.4 \mathrm{~m}$ telescope now under construction on Cerro Pachon in Chile. This ground-based telescope is designed to conduct a decade-long time domain survey of the optical sky. Achieving the fundamental science goals of the LSST requires high quality imaging that is consistent over its full 3.5 degree field of view. LSST will use an Active Optics System (AOS) to achieve this goal. Unlike most other systems, the LSST Active Optics correction will combine an open-loop model with a slow (30 s) closed loop feedback. Although it is not fast enough to correct for atmospheric distortions, the AOS will correct in real time the system aberrations mostly introduced by gravity, temperature gradients and other system distortions. The LSST AOS uses a combination of 4 curvature wavefront sensors (CWS) located on the edge of the LSST field of view. The information coming from the $4 \mathrm{CWSs}$ are combined to calculate the appropriate corrections to be sent to LSST's three mirrors, all equipped with actuators. The AOS software is composed of a Wavefront Estimation Pipeline (WEP) to estimate the error at each of the wavefront sensors and an Active Optics Controller (AOC) to reconstruct the state of the 3-mirrors system and find the optimal correction. In this paper, we describe the design and implementation of the AOS as well as its calibration.
\end{abstract}

Keywords: Active Optics, LSST, curvature sensing, large field of view

\section{INTRODUCTION}

The Large Synoptic Survey Telescope (LSST) is an 8.4 meter diameter telescope now under construction on Cerro Pachon in Chile ${ }^{1,2}$ that will survey the southern sky for 10 years. The particularity of the LSST survey is to be a wide, fast, and deep survey. The LSST telescope will observe 10 square degrees field of view every $39 \mathrm{~s}$ covering over half the sky in two photometric bands every three nights for 10 years. The telescope is composed of three aspheric mirrors: the $8.4 \mathrm{~m}$ primary mirror (M1), a $3.4 \mathrm{~m}$ secondary mirror (M2) and a $5 \mathrm{~m}$ tertiary mirror (M3). The primary and tertiary mirrors form a single monolithic mirror called the M1M3..$^{3,4}$ LSST has only one instrument, a three-lens camera that directs the light path onto a 3.2 gigapixel camera, with a field-of-view (FOV) of 3.5 degrees. ${ }^{5}$ This camera has 6 different filters, $\mathrm{u}, \mathrm{g}, \mathrm{r}, \mathrm{i}, \mathrm{z}, \mathrm{y}$ and is held by a camera hexapod/rotator.

In order to optimize the image quality across the 3.5 degree FOV of the camera, LSST relies on an Active Optics System (AOS). Both M1M3 and M2 are equipped with actuators that allow for the AOS to control the shape of the mirror surface. ${ }^{6}$ In addition, M2 and the camera are mounted on hexapods allowing further positioning adjustment. ${ }^{7}$

The wavefront control is performed using both open-loop measurements (look-up-table) as well as a low temporal frequency closed loop corrections determined from real-time wavefront measurements every $39 \mathrm{~s}$. The open-loop model provides near optimum values for all actuator forces and hexapods positions and depends primarily on elevation angles, azimuth and temperature. These corrections however, do not allow the system to meet the LSST image quality requirements due to non-repeatable and/or unpredictable effects such as temperature uncertainty or hysteresis. The plan is to use real-time wavefront sensor measurements to compensate for these errors. Curvature sensing is used as the wavefront method, since the wide-field and the fast beam make the use of the more popular Shack-Hartmann wavefront sensor problematic. Moreover, because the curvature sensors can be used in the focal plane as opposed to the Shack-Hartmann wavefront sensor, this option minimizes

Further author information: (Send correspondence to S. J. Thomas)

S. J. Thomas: E-mail: sthomas@lsst.org, Telephone: (+1) 520-318-8227 
non-common path errors. More details on the origin and impact of different environmental perturbations on the LSST image quality are described in Angeli et al. ${ }^{8}$

In this paper we describe in more details the architecture and status of the active optics both in term of hardware and software algorithm. Section 2 discusses the wavefront sensing technique. Section 3. and section 4 present the software architecture. Section 5 explains how we will calibrate the wavefront sensor to take into account non-common path between the science sensors and the wavefront sensors.

\section{WAVEFRONT SENSOR}

\subsection{Design}

As mentioned in the introduction, the wavefront sensing technique chosen is curvature sensing. Four wavefront sensors are located at the corner of the focal plane and each sensor is a combination of 2 detectors defocused from each others by $3 \mathrm{~mm}$ (Fig. 1). This allows to take in and out of focus images at the same time simultaneously with the science image. The rate of the wavefront correction is $25.6 \mathrm{mHz}$ (once every $39 \mathrm{~s}$ ) and uses a $15 \mathrm{~s}$ exposure allowing to smooth the atmosphere. It also takes into account previous measurements using Kalman filtering. The size of each defocused image is 7 arcmin by 14 arcmin meaning that there will regularly be numerous donuts to estimate the wavefront (Fig. 1). In addition, we have the capability of defocussing the entire detector in and out of focus using the M2 or the camera hexapods to estimate the wavefront error on the science detectors. The wavefront measurement at the science detector location will allow the calibration of the corner wavefront sensors (see section 5).
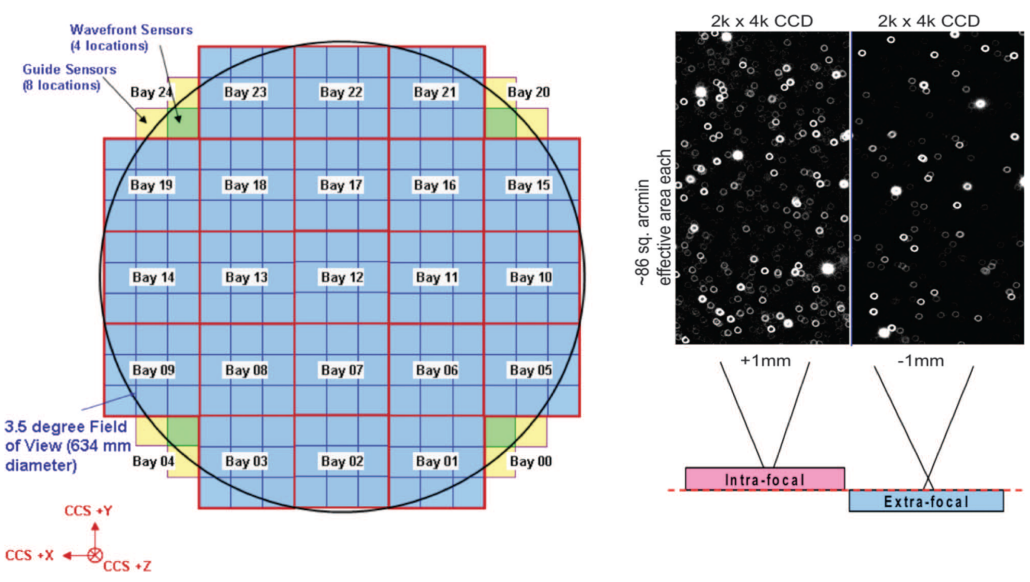

Figure 1. Focal plane layout of the detector. The left drawing shows the full focal plane with the science sensors, the wavefront sensors and the guide sensors. The wavefront sensors and the guide sensors will have vignetting effects as well as distortion that must be taken into account in the wavefront estimation. The right panel shows simulated intra and extra images for one of the wavefront sensors. They were obtained using a complex simulation tool called PhoSim. ${ }^{9}$

\subsection{Physical Offset Estimation}

The optimum relative z-position between the two in and out of focus detectors - or optimum defocus - depends on; atmospheric turbulence, telescope aberrations and irradiance meaning the ability of the AOS to measure aberrations in the system for different atmosphere regime. The advantage of a shorter defocus is to have more irradiance and therefore need a shorter exposure time. Other advantages include the increase of the wavefront sensor sensitivity to low frequency wavefront errors and its improved behaviour in presence of good seeing. The advantages of a longer offset are a higher tolerance of the measurement to aberrations, increased sensitivity to high frequency wavefront errors and higher tolerance to bad seeing.

The optimum defocus also depends on the sky coverage and more specifically the crowding. Indeed, LSST will often observe crowded field and the images will be by nature defocused, creating large donuts difficult to 
isolate. As will be mentioned in section 4.1, the AOS will include a deblending algorithm to take care of the issue. However, the requirement on this algorithm will be eased by adjusting the offset between the detector to decrease the crowding as much as possible without compromising performance.

In order to evaluate what the smallest defocus allowed for the AOS to still perform as required is, a known catalogue and a list of fixed criteria is defined to estimate if a target is a good candidate for wavefront sensing. The catalog chosen is the SST-RC5 catalogue ${ }^{10}$ which is a compilation of multiple surveys such as 2MASS, SDSS etc. Because of the incompleteness of the catalogue, the filters used are G, R and I bands. The sky is divided into 2782 fields for which the detectors are aligned with the RA and Dec grid on the sky. The list of criteria includes:

a. The location of the stars. The location has to match the observable sky coverage of LSST and is included in a area defined by a right ascension from 0 to $360 \mathrm{deg}$, a declination between -90 and +4 as well as a crescent defined by a right ascension from 0 to $12 \mathrm{deg}$ and a declination between +0 and +32 .

b. The magnitude limit. The magnitude limit is set by the signal to noise ratio per pixel (SNR $=10)$. Because the SNR is per pixel and because this study assumes only one star is assumed to create the master donut, the results give a conservative estimation of the sky coverage.

\section{c. The detector saturation limit}

d. The nature of the neighbors within 2.5 times the radius of the defocused donut around each of the targets satisfying points a to c above. A target with no neighbors is a target that has no stars in within this area or a star dimmer than the range of magnitude for a given filter. The neighbors were classified into 3 different categories: (i) neighbors with a magnitude lower than the saturation limit given by c); (ii) neighbors with magnitudes within the range given by b) and c); (iii) neighbors with magnitudes larger than the minimum magnitude given by c).

As expected, the shorter the wavelength the least sensitive to crowding the AOS is. This study allows us to define the optimal detector separation. Studies have shown that it is also possible to use 3 out of the 4 wavefront sensors, which also gives more flexibility.

Note that it is expected that the $\mathrm{U}$ band will be limited by target availability (favoring shorter offsets as the SNR increases when decreasing the offset). For $\mathrm{Y}$ and $\mathrm{Z}$ band, we are expecting the crowding to get worse and therefore the requirements on the deblending algorithm to become more stringent.

\section{CORRECTIVE SYSTEM}

The LSST optical system utilizes a unique, compact, three-mirror design consisting of an 8.4-meter primary mirror (M1), 3.5-meter secondary mirror (M2), and a 5.0-meter tertiary mirror (M3) .

The Primary/Tertiary mirror system is a monolith M1M3 sharing the same single cast borosilicate substrate, which improves the stiffness (Fig. 2). M3 resides within the 5-meter diameter central hole of the primary mirror assuring a permanent orientation of the M3 toward the M1. Such a configuration does simplifies optical alignment and correction of misalignment by removing six degrees of freedom. The mirror started fabrication in July of 2008 by the Mirror Laboratory at the University of Arizona Tucson and was polished, inspected and accepted by LSST in April 2015. ${ }^{3}$ The mirror cell was fabricated by CAID in Tucson and is undergoing acceptance tests. The integration of the actuators and final tests with the mirror is scheduled later this fall and beginning of 2018.

The secondary mirror is a 3.4-meter diameter convex mirror from a $100 \mathrm{~mm}$ thick meniscus ULE blank completed by Corning Incorporated in 2009 (see Fig. 3-left). The mirror is undergoing ion figuring and polishing by Harris Corporation (formerly Exelis) in Rochester. Harris Corporation also provides the mirror cell with its active control (Fig. 3-right). The measurement of the entire optical surface was made challenging by the convex shape of the optics and Harris was required to develop a novel technique of sub-aperture stitching for this purpose. More information on the results will be presented in later papers. 

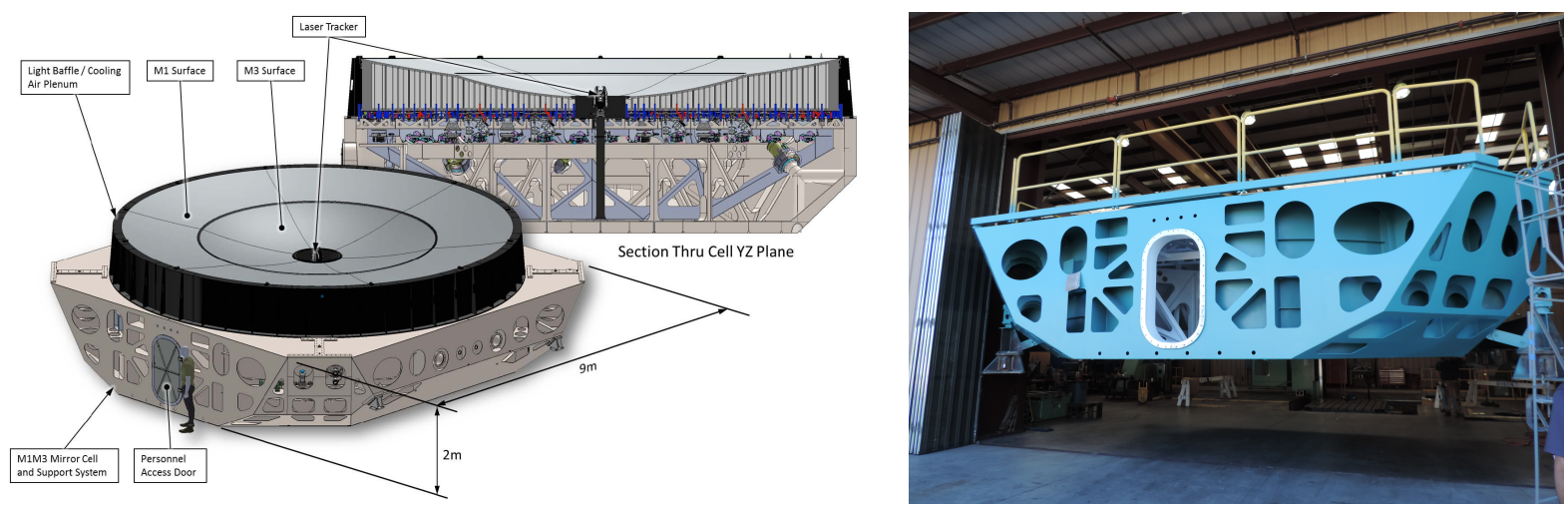

Figure 2. Left: cartoon of the mirror cell showing the location of the main elements. Right: Real cell at CAID in Tucson in September 2017
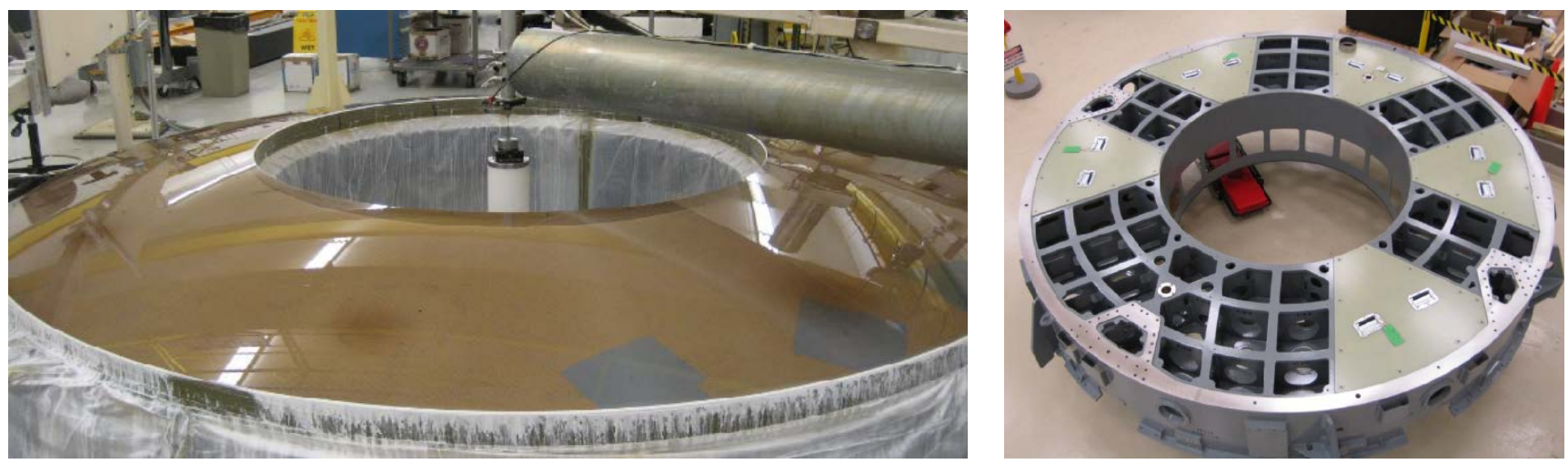

Figure 3. Left: Mirror M2, a 3.4m convex mirror. Right: Mirror cell

The optical system possesses several degrees of freedom to control the shapes of M1M3 and M2 as well as to control the camera and M2 x/y decenter and x/y tilt. M1M3 serves as a reference of the full telescope.

During observing, the M1M3 mirror is actively supported by 156 pneumatic figure control actuators (see Fig. 4). These figure control actuators resist loads (gravitational, wind, dynamic, etc.) and provide the active optics closed loop figure control. The position of the mirror relative to the mirror cell is controlled by a set of six hardpoints (displacement controlled actuators) that form a large hexapod.

The mirror also supports a robust thermal control system to reduce the thermal deformations of the mirror and to reduce the convection from the mirror's optical surface, which can degrade significantly the final image quality. This is even more important since, as mentioned earlier, the mirror M1M3 is made of borosilicate glass that has a large coefficient of thermal expansion. ${ }^{4}$ The thermal control system consists of 96 fan blower units which circulate conditioned air via 1800 nozzles to vent the mirror cores.

Similarly to M1M3, during observing, the mirror M2 is supported by 72 axial actuators and 6 tangent links. Because the coefficient of thermal expansion is not as large as the one for M1M3, the mirror M2 does not require a thermal control system.

Both mirrors will take into account 20 bending modes for closed loop correction.

Finally, there will be a thermal compensation for the telescope mount to minimize the tube elongation of the structure. Indeed, the required uncertainty if the temperature measurements for such a fast telescope is quite challenging. For instance the thermal expansion coefficient of steel is about $12^{-6}$ per degree Celcius, and the tube is roughly 8 meters long, consequently the actual thermal expansion amounts to approximately $96 \mathrm{mum}$ per degree Celcius C. To be able to compensate for displacements in the order of single microns the temperature 

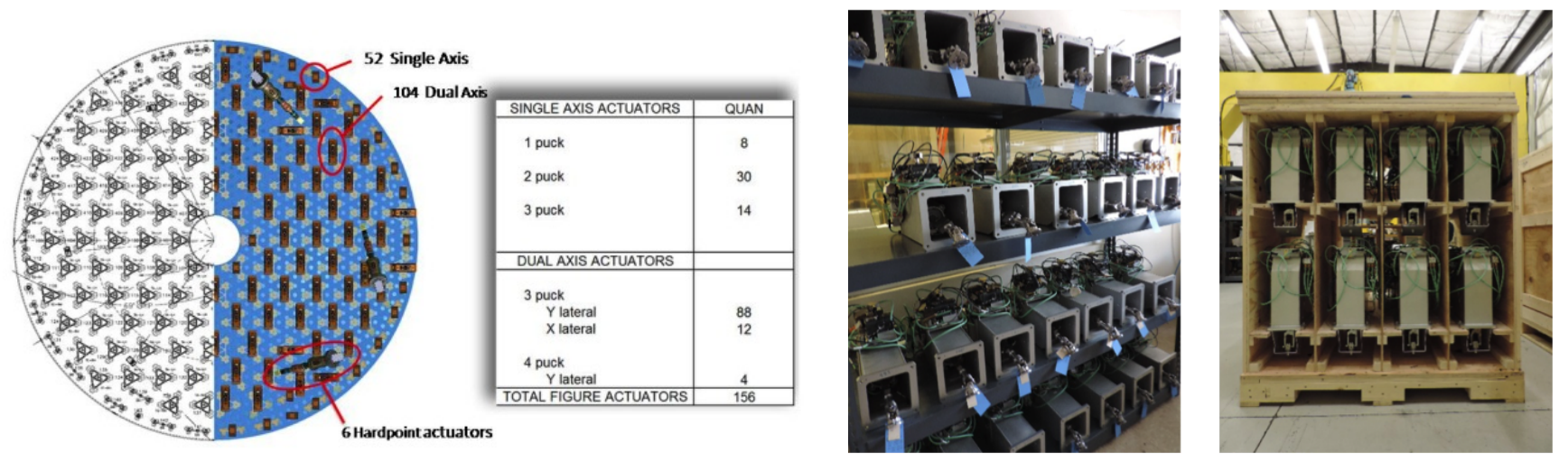

Figure 4. Left: Location of the different actuators. Middle: Single axis actuators built by LSST. Right: Dual axis actuator built by LSST.

measurement system (sensor + instrument) must be capable of delivering uncertainties better than $0.0017 \mathrm{C}$ RMS.

\section{AOS SOFTWARE}

The AOS software is mainly composed of (i) the Wavefront Estimation Pipeline (WEP) (ii) the Active Optics Controller (AOC) and finally (iii) the mirrors and hexapods controllers (see Fig. 5.) Most of this section describes the first two parts of the software. Fig. 5 shows the main architecture of the AOS. The Observatory Control System (OCS) coordinates the different main controllers such as the Camera Control System (CCS), the Data Management Control System (DMCS), the Telescope Control System (TCS) to respectively take AOS images, save AOS images and process AOS images. Be aware that these responsibilities are different for the science detector images. The WEP, AOC and other mirror/hexapods controllers belong to the TCS. The following section will explain these two main components and the interfaces they require.

\subsection{Wavefront Estimation Pipeline}

The Wavefront Estimation Pipeline (WEP) is responsible for estimating the wavefront errors in annular Zernike coefficients associated with each of the corner rafts and each science detector if needed. In regular nightly operation, the inputs to the WEP are an intra-focal image and an extra-focal image coming from each of the four corner rafts as described in section 2. For calibration purposes, the inputs are images from any detectors in the science focal plane taken after sequently moving the whole focal plane in and out of focus using the hexapods. The software architecture is show in Fig. 6

Because of its design, LSST requires a special adaptation of the regular curvature sensing algorithm used so far in adaptive optics. Indeed, LSST has a very fast beam, and because of the location of the corner rafts in the focal plane (1.7 degrees off-axis) the WFS detectors see a lot of distortion and vignetting that need to be taken care of before analyzing the images. Another difference with typical curvature sensing seen in adaptive optics is the number and nature of the stars. Each of the in and out of focus detector of a particular corner raft has a large FOV $-7 \times 14$ arcmin - and sees a different field of the sky. Therefore there are numerous stars to use for wavefront sensing and the stars on each the 2 detectors in each corner are different. Finally, LSST has a large central obstruction of $60 \%$. More information on the method used to compensate for these particular effects can be found in Xin et al. ${ }^{11,12}$

After being processed by the Wavefront Data Collector (WF Data Collector), the images are corrected from basic instrument signature using a procedure called the Instrumentation Signature Removal (ISR) ${ }^{13,14}$ developed by the Data Management team. The ISR algorithm includes typical astronomical calibration (Flat fielding, bad pixels, darks, biases, gains). The next step is the source selector, defining which source is a good candidate for WEP. Indeed, because of the large FoV, there are numerous options and the algorithm uses a catalogue to simplify the process. The source selector will identify targets from a bright star catalog such as the one from the SST-RC5 


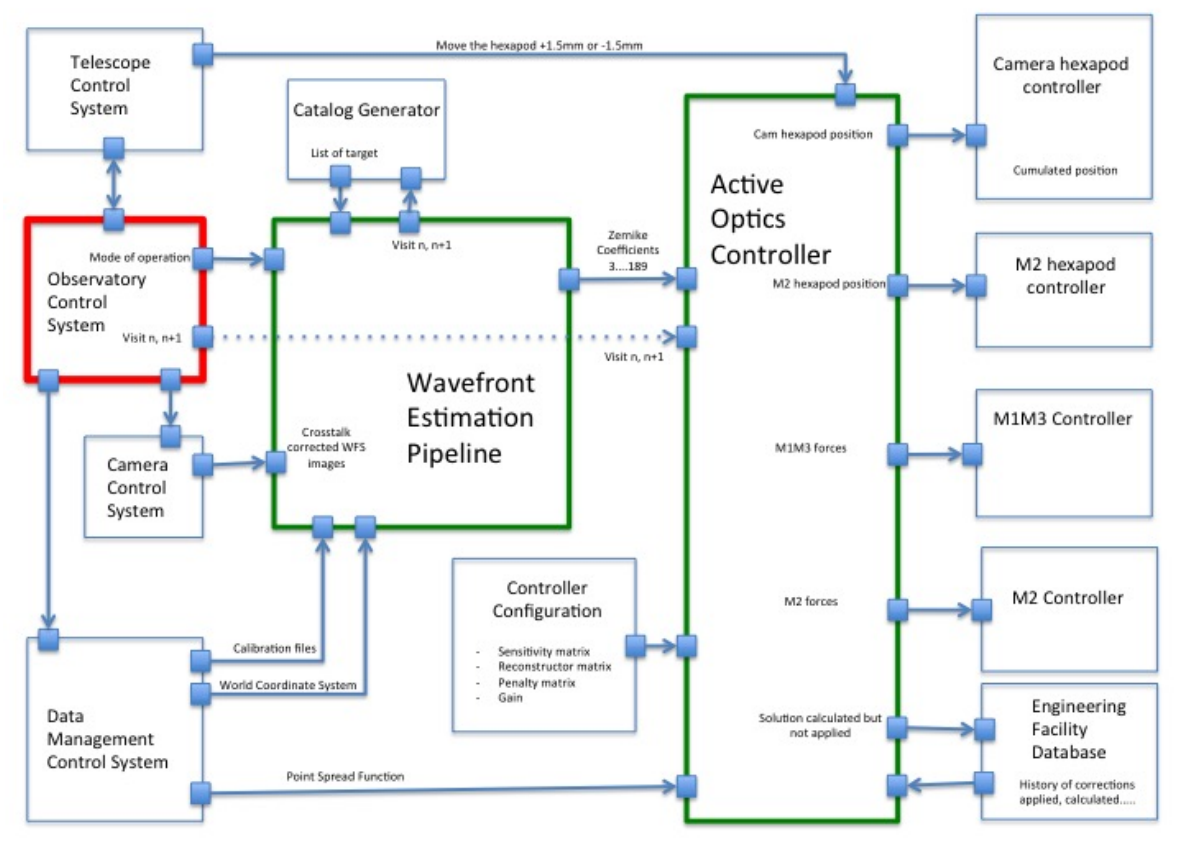

Figure 5. Active Optics System overarching block diagram. The list of acronyms is given in the main text.

catalog, ${ }^{10}$ Gaia ${ }^{15}$ and ultimately LSST own catalog to be used in the donut selection to estimate the wavefront error. The target selection criteria include the magnitude of the star as well as the number and brightness of its neighbors (section 2.2). Indeed, because the AOS is dealing with defocussed images, the wavefront sensors will more sensitive to crowding than focused images. A compromise for the offset between the two detector in a corner raft was made looking at the sky coverage, as described in section 2.2. Once the target is selected, each source of a field is deblended from potential neighbors and the intensity scaled to unity. Finally an in/out master image is created and sent to the wavefront estimator itself. The output of the wavefront estimation pipeline is annular Zernike for each detector used. Another component is the wavefront estimator configurator used to set up input to the wavefront estimator. All the important data will be saved in a queryable database called the Engineering Facility Database (EFD).

\subsection{Active Optics Controller}

The Active Optics Controller is the overall optical feedback algorithm called the active optics controller and can be separated into two steps: the Optical State Estimator and the Optimal Controller (Fig. 7). The appropriate optical state is derived from the wavefront sensors in the presence of algorithmic and atmospheric noise errors. Also included in the AOC is a Kalman filtering algorithm using the history of either the previous measured Zernike Coefficients, the optical state or the control commands.

The method to calculate the commands $u$ from the measurements $y$ is as follow. Once can write $y=A x+w$, where $A$ is the sensitivity matrix, $w$ the noise and $x$ is the optical state. The noise being quite small, we can write the minimum variance estimate of $x$, called the optimal optical state $\hat{x}$ as a function of the pseudo-inverse of the $A$ matrix, $X$ is the covariance of the optical state and the noise covariance matrix $W$ :

$$
\hat{x}=X A^{T}\left(A X A^{T}+W\right)^{-1} y
$$

The measurement $y$ is vector of Nx20 Zernike coefficients measured by the WEP for each wavefront or science sensors. The value for $\mathrm{N}$ ranges from 3 to 189 depending on the use case. The optical state $x$ is a vector with the 22 bending modes for each mirrors and the 6 values for the M2 and Camera rigid body positions. The 


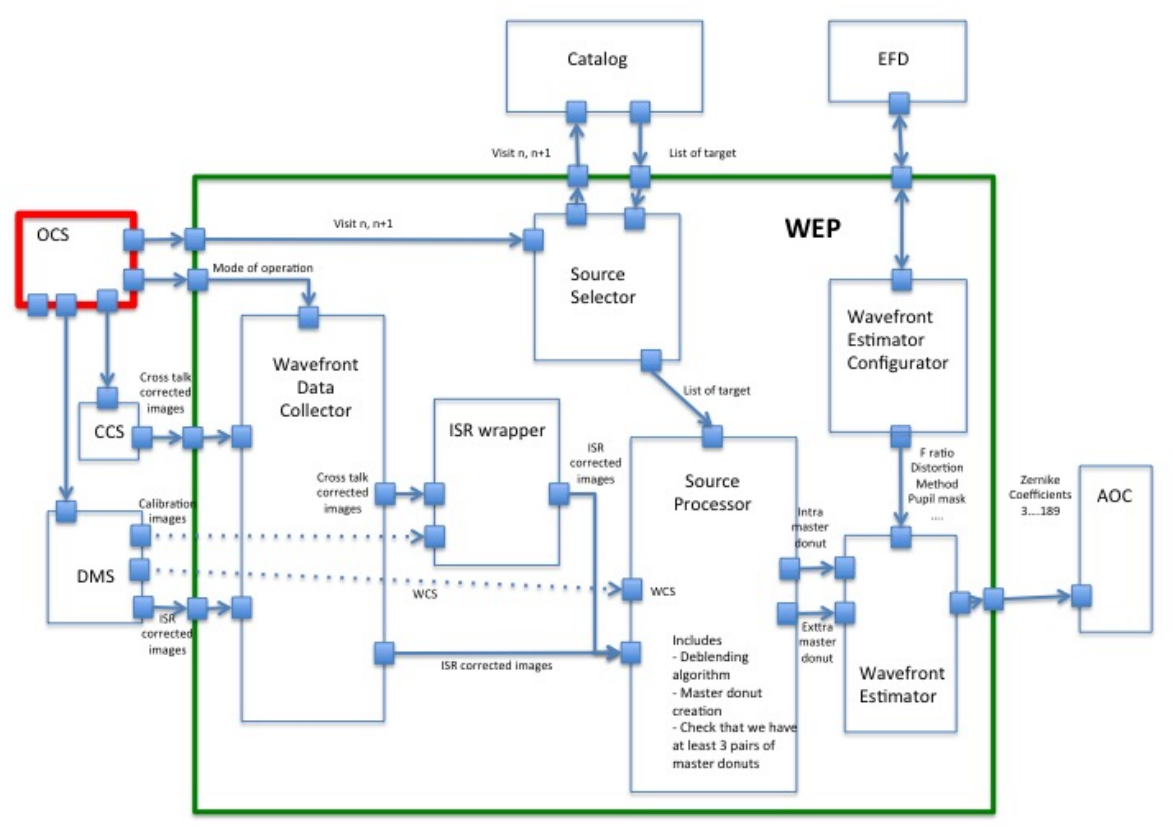

Figure 6. Wavefront Estimation Pipeline (WEP). The list of acronyms is given in the main text.

dimensions for the sensitivity matrix $A$ is driven by the sizes of $x$ and $y$. From this optimized optical state, an optimal control algorithm (see Fig. 8) utilizes a cost function $J$ to derive the realistic coefficients $u$ for the bending modes offsets that need to be applied to M1M3 and M2 shape and the positions for the Camera and M2 hexapods. The cost function is built to optimize the image quality, limit large swings to avoid damage to the glass and ensure smooth transition between iterations. This cost function includes a penalty matrix $(H)$, a penalty factor $(\rho)$ and a quality matrix $(Q)$ such that:

$$
J=x^{T} Q x+\rho u^{T} H u
$$

The penalty matrix $H$, is used to minimize the near-degeneracy in the $A$ matrix can result in large excursions in individual mirror commands since the control will command any displacement value if that value results in a perceived reduction in FWHM, no matter how small. In other term, it allows the control of where the solution should be considered (M1, M2 or M3) mostly due to glass safety and other potential inputs such as near-degeneracy.

\section{ACTIVE OPTICS SYSTEM CALIBRATION}

The LSST field of view is very large (10 square deg) and the wavefront sensors are located at the corner of the field leading to potential non-common path errors between the center detectors and the wavefront sensors. Similarly to many wavefront sensors, there is a need to calibrate the solution in order to obtain the best image quality for the science detectors. To calibrate the offsets between the science sensors and the WFS, the wavefront error will be measured by curvature sensing at the center of each science detector by defocusing the camera using the camera's hexapod. The images are then analyzed using the same pipeline as for the four corner wavefront sensors. The difference of solution represents the offsets between the science detector and the wfs and will be incorporated in the the open-loop-model. This offset depends on the elevation and azimuth angles. This calibration process is under study and more development will occur in the following year with an eye on commissioning activities.

Another method under study is to look at the Point Spread Function (PSF) at each detector to estimate the variation of the correction across the field as described in Davis et al. ${ }^{16}$ 


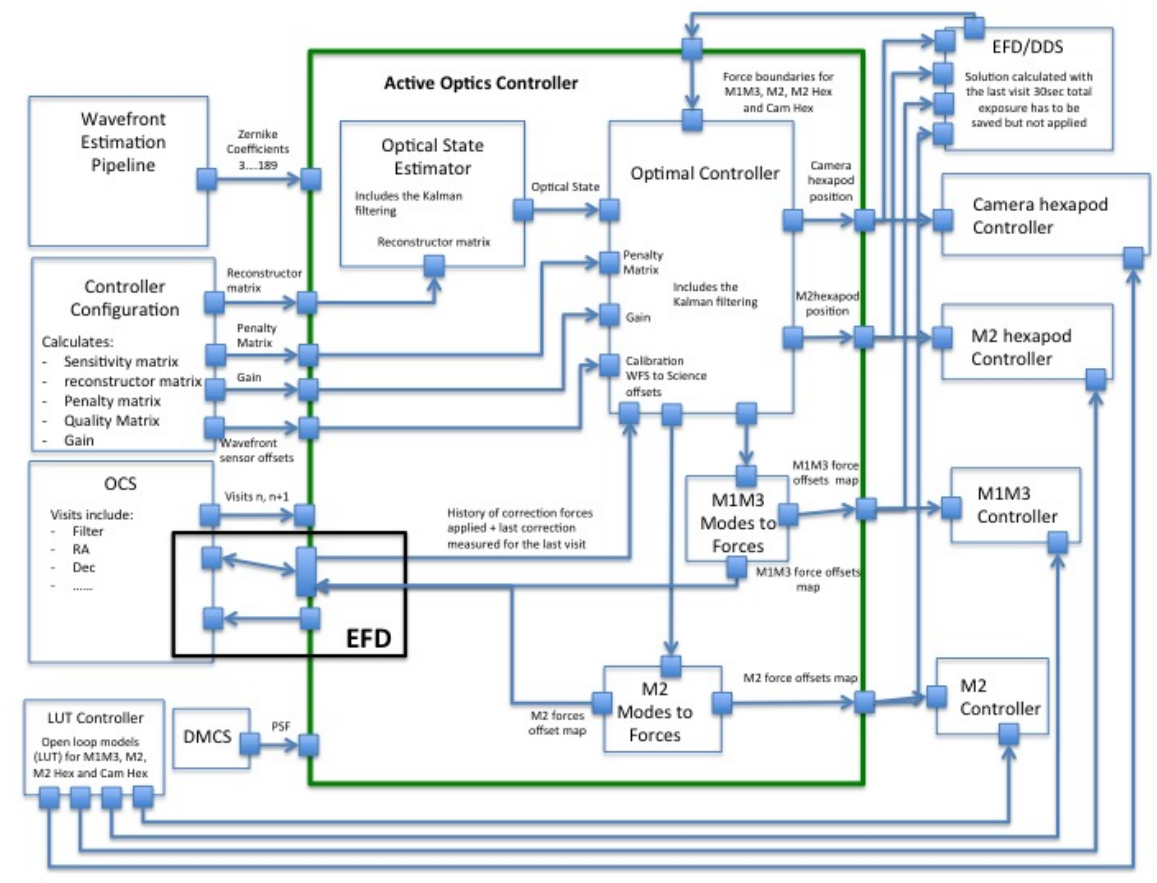

Figure 7. Block diagrams of the active optics controller. The list of acronyms is given in the main text.

\section{CONCLUSION}

The LSST Active Optics System is complex considering the telescope particular design and the number of degrees of freedom in the system. In this paper we presented the status of the AOS design both for the hardware and the software elements. Knowledge gathered in the process will be useful for future Extremely Telescopes. Indeed, even though the goal is not to correct for the atmospheric turbulence, lesson learnt regarding the M1M3 mirror will be useful for the Giant Magellan Telescope project and the experience gained using the M2 mirror would be beneficial for extremely large telescope's adaptive secondary mirrors in general. First light and first commissioning tests of the LSST AOS is planned for 2020.

\section{ACKNOWLEDGMENTS}

This material is based upon work supported in part by the National Science Foundation through Cooperative Agreement 1258333 managed by the Association of Universities for Research in Astronomy (AURA), and the Department of Energy under Contract No. DE-AC02-76SF00515 with the SLAC National Accelerator Laboratory. Additional LSST funding comes from private donations, grants to universities, and in-kind support from LSSTC Institutional Members.

\section{REFERENCES}

[1] Ivezic, Z., Tyson, J. A., and et al., for the LSST Collaboration, "LSST: from Science Drivers to Reference Design and Anticipated Data Products," ArXiv e-prints (May 2008).

[2] Gressler, W. J., "LSST telescope and site status," in [Ground-based and Airborne Telescopes VI], Proc. SPIE 9906, 99060J (July 2016).

[3] Araujo-Hauck, C., Sebag, J., Liang, M., Neill, D., Muller, G., Thomas, S. J., Vucina, T., and Gressler, W. J., "LSST mirror system status: from design to fabrication and integration," in [Ground-based and Airborne Telescopes VI], Proc. SPIE 9906, 99060L (July 2016). 


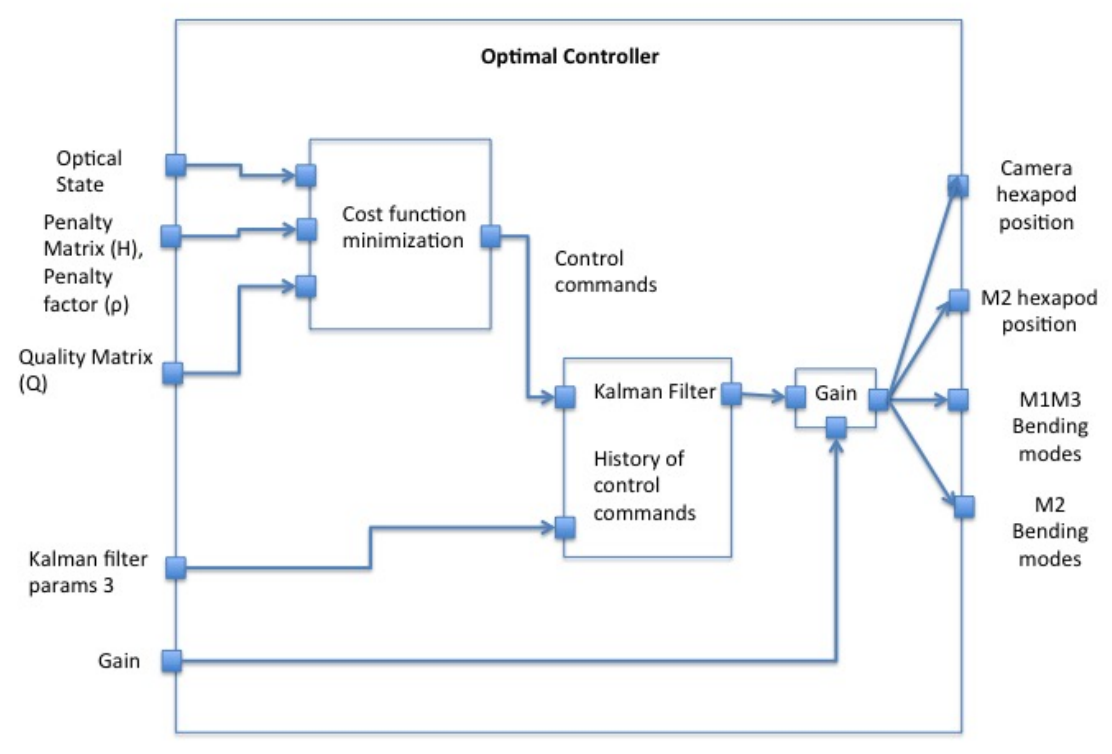

Figure 8. Block diagrams of the optimal controller embedded in the Active Optics Controller.

[4] Neill, D. R., Bogan, G., Zajac, D., Araujo, C., Gressler, W. J., DeVries, J., Hileman, E. A., Lotz, P. J., Mills, D., Thomas, S., Sebring, T. A., Sebag, J., Warner, M., and Wiecha, O., "LSST secondary mirror system final design," in [Ground-based and Airborne Telescopes VI], Proc. SPIE 9906, 990667 (July 2016).

[5] Kahn, S. M., Kurita, N., Gilmore, K., Nordby, M., O'Connor, P., Schindler, R., Oliver, J., Van Berg, R., Olivier, S., Riot, V., Antilogus, P., Schalk, T., Huffer, M., Bowden, G., Singal, J., and Foss, M., "Design and development of the 3.2 gigapixel camera for the Large Synoptic Survey Telescope," in [Ground-based and Airborne Instrumentation for Astronomy III], Proc. SPIE 7735, 77350J (July 2010).

[6] Neill, D., Angeli, G., Claver, C., Hileman, E., DeVries, J., Sebag, J., and Xin, B., "Overview of the LSST active optics system," Proc. SPIE 9150, 91500G-91500G-16 (2014).

[7] Sneed, R., Neill, D. R., Kidney, S., Araujo, C., Gressler, W., Lotz, P. J., Milles, D., Sebag, J., Sebring, T. A., Warner, M., and Wiecha, O., "Final design of the LSST hexapods and rotator," in [Ground-based and Airborne Telescopes VI], Proc. SPIE 9906, 99060K (July 2016).

[8] Angeli, G. Z., Xin, B., Claver, C., MacMartin, D., Neill, D., Britton, M., Sebag, J., and Chandrasekharan, S., "Real time wavefront control system for the Large Synoptic Survey Telescope (LSST)," Proc. SPIE 9150, 91500H-91500H-16 (2014).

[9] Peterson, J. R., Jernigan, J. G., Kahn, S. M., Rasmussen, A. P., Peng, E., Ahmad, Z., Bankert, J., Chang, C., Claver, C., Gilmore, D. K., Grace, E., Hannel, M., Hodge, M., Lorenz, S., Lupu, A., Meert, A., Nagarajan, S., Todd, N., Winans, A., and Young, M., "Simulation of astronomical images from optical survey telescopes using a comprehensive photon monte carlo approach," The Astrophysical Journal Supplement Series 218(1), $14(2015)$.

[10] Monet, D. private communication (2016).

[11] Xin, B., Claver, C., Liang, M., Chandrasekharan, S., Angeli, G., and Shipsey, I., "Curvature wavefront sensing for the Large Synoptic Survey Telescope," Applied Optics 54, 9045- (Oct. 2015).

[12] Xin, B., Roodman, A., Angeli, G., Claver, C., and Thomas, S., "Comparison of LSST and DECam wavefront recovery algorithms," in [Ground-based and Airborne Telescopes VI], Proc. SPIE 9906, 99064J (July 2016).

[13] Jenness, T., Bosch, J., Owen, R., Parejko, J., Sick, J., Swinbank, J., de Val-Borro, M., Dubois-Felsmann, G., Lim, K.-T., Lupton, R. H., Schellart, P., Krughoff, K. S., and Tollerud, E. J., "Investigating interoperability 
of the LSST data management software stack with Astropy," in [Software and Cyberinfrastructure for Astronomy IV], Proc. SPIE 9913, 99130G (July 2016).

[14] Jurić, M., Kantor, J., Lim, K.-T., Lupton, R. H., Dubois-Felsmann, G., Jenness, T., Axelrod, T. S., Aleksić, J., Allsman, R. A., AlSayyad, Y., Alt, J., Armstrong, R., Basney, J., Becker, A. C., Becla, J., Bickerton, S. J., Biswas, R., Bosch, J., Boutigny, D., Carrasco Kind, M., Ciardi, D. R., Connolly, A. J., Daniel, S. F., Daues, G. E., Economou, F., Chiang, H.-F., Fausti, A., Fisher-Levine, M., Freemon, D. M., Gee, P., Gris, P., Hernandez, F., Hoblitt, J., Ivezić, Ž., Jammes, F., Jevremović, D., Jones, R. L., Bryce Kalmbach, J., Kasliwal, V. P., Krughoff, K. S., Lang, D., Lurie, J., Lust, N. B., Mullally, F., MacArthur, L. A., Melchior, P., Moeyens, J., Nidever, D. L., Owen, Russell aNeillnd Parejko, J. K., Peterson, J. M., Petravick, D., Pietrowicz, S. R., Price, P. A., Reiss, D. J., Shaw, R. A., Sick, J., Slater, C. T., Strauss, M. A., Sullivan, I. S., Swinbank, J. D., Van Dyk, S., Vujčić, V., Withers, A., Yoachim, P., and LSST Project, f. t., "The LSST Data Management System," Astronomical Data Analysis Software and Systems XXV proc., arXiv:1512.07914 (2015).

[15] Gaia Collaboration, Prusti, T., de Bruijne, J. H. J., Brown, A. G. A., Vallenari, A., Babusiaux, C., BailerJones, C. A. L., Bastian, U., Biermann, M., Evans, D. W., and et al., "The Gaia mission," A\&A 595, A1 (Nov. 2016).

[16] Davis, C. P., Rodriguez, J., and Roodman, A., "Wavefront-based PSF estimation," in [Ground-based and Airborne Telescopes VI], Proc. SPIE 9906, 990668 (July 2016). 